\title{
Treatment with Radiopharmaceuticals and Radionuclides in Breast Cancer: Current Options
}

\author{
Burcu Altıparmak Güleç ${ }^{1}$, (1) Fatma Yurt² \\ ${ }^{1}$ Department of Nuclear Applications, Institute of Nuclear Science, Ege University, İzmir, Turkey (Graduated) \\ ${ }^{2}$ Department of Nuclear Applications, Institute of Nuclear Science, Ege University, İzmir, Turkey
}

\begin{abstract}
Radiopharmaceutical therapy (RPT) is an effective and safe treatment for many types of cancer. RPT acts by binding radioactive atoms to tumor-associated antigens, monoclonal antibodies, nanoparticles, peptides, and small molecules. These treatments ensure that a concentrated dose is delivered to the targeted tumor tissue while preserving the normal tissues surrounding the tumor. Given these features, RPT is superior to traditional methods. This review article aimed to performa comprehensive review and evaluation of the potential of radionuclides and radiopharmaceuticals used in breast cancer treatment in preclinical studies conducted in the last five years.
\end{abstract}

Keywords: Breast cancer, radioisotopes, radionuclide therapy, radiopharmaceuticals

Cite this article as: Altıparmak Güleç B, Yurt F. Treatment with Radiopharmaceuticals and Radionuclides in Breast Cancer: Current Options. Eur J Breast Health 2021; 17(3): 214-219

\section{Key Points}

- Radiopharmaceutical therapy is a safe and effective method for cancer treatment.

- Radiopharmaceutical therapies have minimal toxicity compared to other cancer treatments, making the option successful and useful.

- The design of radiopharmaceuticals requires various pharmacokinetic and biofunctional procedures, and the selection of the appropriate method increases specificity and provides a successful treatment method.

- For the in vitro and in vivo experiments to be performed comprehensively, conditions in the limited competition environment must be provided equally in terms of cost and quality.

- According to breast cancer types, increasing specific radiopharmaceutical designs in breast cancer treatment has increased the probability of success.

\section{Introduction}

The World Health Organization (WHO) reported that 18.1 million people were diagnosed with cancer in 2018 and 9.6 million people died in the same year. With the increase in diagnostic modalities, more people are being diagnosed with cancer, and the ensuing public health problem has led to the investigation of more treatment options in this field. Worldwide, the most common types of cancer in men are lung, prostate, stomach, and liver cancers, where as the most common types in women are breast, lung, cervical, and colon cancers. In a 2018 WHO report, it was stated that 2.09 million women were diagnosed with breast cancer that year. Attention is drawn to the importance of new treatment options considering this rate and the fact that breast cancer is the most common type of cancer in women $(1,2)$. The application of radioisotope and radiopharmaceutical therapies in many cancer treatments [thyroid (3), gastrointestinal system (4), prostate (5), gastroenteropancreatic, lung (6), non-Hodgkin's lymphoma (7), cancers such as prostate bone metastases (8), breast bone metastases (9)] and positive results obtained have directed researchers to preclinical studies about the applicability of these treatment methods to breast cancer.

These treatments aim to target cancerous cells directly and protect healthy tissues. Radionuclide therapy (RT) involves sending radioactive elements with different emission properties into cancerous tissue. RT has the advantage of delivering a highly concentrated dose to the targeted tumor tissue while protecting the normal tissues surrounding the tumor. The fact that these treatment practices are minimally invasive, and the duration of treatment is shorter than that of chemotherapy has made them one of the most preferred cancer treatment methods recently (10). 
Radiopharmaceutical therapy (RPT) is carried out by sending radionuclides conjugated to tumor-targeted pharmaceuticals or bioactive molecules (nanoparticles, antibodies, peptides, small molecules, and various structures of each) that predominantly accumulate in neoplastic cells within tumors into the tumor tissue (11). Recently, RT has attracted attention as a safe and effective method for cancer treatment, and research continues (12).

In this review article, the effectiveness of preclinical studies conducted in the last five years with radionuclides and radiopharmaceuticals in breast cancer will be discussed.

\section{Therapeutic radionuclides used as radiopharmaceuticals History of radiopharmaceuticals}

To understand RPT, we first need to talk about radioactive atoms and their history. In 1930, Rutherford mentioned three important discoveries in the publications of Chadwick and Ellis named "Radiation from radioactive materials". These were the discovery of X-rays by Roentgen in 1895, the discovery of the radioactivity of uranium by Becquerel in 1896, and the discovery of the electron in 1897 by J.J.Thomson. Briefly, the discovery of atomic physics went through the following stages (13). Radiography was not discovered until 1895, when Wilhelm Conrad Roentgen was exposed totoX-rays by chance while working in the laboratory with a cathode ray (X-ray) tube. Shortly after the discovery of X-rays, French scientist Henri Becquerel found that uranium mineral fluoresces when exposed to sunlight, and in 1903 Marie and Pierre Curie shared the Nobel Prize in Physics with this discovery. Subsequently, Henri Becquerel and Marie Curie worked together to discover radioactive elements other than uranium, including radium and polonium. Curie believed that radium could be used to relieve pain (14).

According to the history of radiation in medicine, in 1901, Becquerel noticed burning caused by radium he carried in his pocket, which made him realize diseases were caused by radioactive materials. Henri Alexander Danlos and Eugene Block placed radium in contact with a skin lesion and initiated the first use of RT. Alexander Graham Bell suggested placing radium sources in and near tumors in 1903 and published Frederick Proesher's first study with intravenous injection of radium in 1913 (10). Radium beams were used in certain diseases, including systemic lupus, cancer, and nerve diseases (14). The use of radiation for therapeutic purposes dates back this far.

In recent years, although radiopharmaceuticals containing particleemitting radionuclides such as ${ }^{90} \mathrm{Y}$-ibritumomab tiuxetan (Zevalin), strontium-89 chloride (Metatron), and iobenguane ( ${ }^{131}$ I-Azedra) were once preferred in therapeutic radiopharmaceutical research, radionuclides that emit alpha particles have now become the focus of attention. In 1997, the US Food and Drug Administration (FDA) approved the ${ }^{213} \mathrm{Bi}$ radionuclide for use in clinical trials. The first radiopharmaceutical approved by the US-FDA in 2013 was ${ }^{223} \mathrm{RaCl}_{2}$ (15).

\section{Physical and biological efficacy of therapeutic radionuclides}

To understand RPT, it is first necessary to understand what type of ionizing radiation is emitted by the radioisotopes used in these compounds. There are three different types of ionizing radiation: photons, electrons, and alpha particles. Photons are made up of X-rays and $\gamma$-rays, and X-rays have lower energy than $\gamma$-rays. Photon emissions are useful for monitoring responses to therapy after treatment but are not suitable for localizing cytotoxic radiation in tumor cells (16). Therefore, the preferred ionizing radiation types for the treatment of tumor tissue are electrons and alpha particles. Alpha particles have a high linear energy and a short range; beta particles have a low linear energy and a long range (17).

For RPT to be effective, the distance these particles reach, the amount of energy they accumulate in cells, and direct interaction with DNA are of great importance. Beta particles form free radicals by breaking the covalent bonds of water molecules in tissues, and these radicals cause DNA damage by causing double-strand breakage. When these two particles are compared to each other based on double-stranded DNA breakage, cell death requires one alpha particle or hundreds of beta particles. The high linear energy and short range of alpha particles in the tissue ensure that toxicity around the tumor tissue is low, and the cancer cells sustain a high level of damage. Therefore, it is considered that the cytotoxicity of alpha particles is much higher than thatof beta particles (8). Radiopharmaceuticals have been used in diagnostics and therapeutics for more than half a century. Compared with other cancer treatment options, RPT has minimal toxicity. The features of an ideal RPT include aspects such as good radionuclide radiation type (alpha, beta, and auger electron) selection, radiation energy higher than $1 \mathrm{MeV}$, effective half-life in hours or days, high target tissue ratio/ non-target tissue ratio, low cost, and ease of procurement, and ease of preparation in the laboratory (18). The remarkable potential of RPT against primary and long-distance metastases has led to its acceptance as an appropriate treatment method because it is effective, safe, and economical (16).

\section{Selection of radionuclides for therapeutic purposes}

In nuclear medicine, $95 \%$ of radiopharmaceuticals are used for diagnosis and 5\% for treatment. Radionuclides are divided into two types: diagnostic and treatment radionuclides. The most common and ideal radionuclide used for diagnosis is ${ }^{99 \mathrm{~m}} \mathrm{Tc}$. The reason for the preference of this radionuclide in diagnosis is that it emits pure gamma radiation with an energy between 100 and $250 \mathrm{keV}$; is easily available and affordable; has ane ffective half-life; has a high target/ non-target ratio; and is sterile, pyrogenic, isotonic, and isohydric. Imaging radionuclides used other than ${ }^{99 \mathrm{~m}}$ Tcincludefluorine-18 $\left({ }^{18} \mathrm{~F}\right)$, carbon-11 $\left({ }^{11} \mathrm{C}\right)$, nitrogen- $13\left({ }^{13} \mathrm{~N}\right)$, oxygen- $15\left({ }^{15} \mathrm{O}\right)$, and gallium-68 $\left({ }^{68} \mathrm{Ga}\right)$. For nearly 50 years, ${ }^{131} \mathrm{I}$ was used for the treatment of thyroid cancer (19). Apart from this, some beta radionuclides used for therapeutic purposesinclude ${ }^{153} \mathrm{Sm},{ }^{177} \mathrm{Lu}, \mathrm{Y}-90$, and ${ }^{131} \mathrm{I}$, along with the alpha radionuclides ${ }^{211} \mathrm{As},{ }^{212} \mathrm{Bi},{ }^{212} \mathrm{~Pb},{ }^{213} \mathrm{Bi},{ }^{225} \mathrm{Ac},{ }^{223} \mathrm{Ra}$, and ${ }^{227} \mathrm{Th}$. The physical properties of some therapeutic radionuclides are given in Table $1(16,17,20)$.

When choosing a therapeutic radionuclide, attention should be paid to its physical and biochemical properties. Physical properties include physical half-life, types of emission, the energy of radiation(s), by-product(s), method of production, and radionuclide purity. Biochemical properties include tissue targeting, retention of radioactivity in the tumor, in vivo stability, and toxicity (21). When choosing the treatment radionuclide, attention should be paid to its effective half-life. The effective half-life is calculated from the biological and physical half-life in the body or organs. The effective half-life $\left(\mathrm{T}_{\mathrm{e}}\right)$ is calculated by $\mathrm{T}_{\mathrm{e}}=\mathrm{T}_{\mathrm{p}} \cdot \mathrm{T}_{\mathrm{b}} /\left(\mathrm{T}_{\mathrm{p}}+\mathrm{T}_{\mathrm{b}}\right)$. The physical half-life $\left(\mathrm{T}_{\mathrm{p}}\right)$ is included in the literature for each radionuclide, but the biological half-life $\left(\mathrm{T}_{\mathrm{b}}\right)$ requires the biodistribution of the radiopharmaceutical 
Table 1. Therapeutic Radionuclides $(16,17,20)$

\begin{tabular}{cccc}
\hline Radionuclides & Mode of decay & Physical half-life $\left(\mathbf{t}_{1 / 2}\right)$ & $\mathbf{E}_{\text {max }}(\mathbf{M e V})$ \\
\hline${ }^{90} \mathrm{Y}$ & $\beta$ & 64.10 hours & 0.3 \\
${ }^{131} \mathrm{I}$ & $\beta^{-}$ & 8.02 days & 0.5 \\
${ }^{177} \mathrm{Lu}$ & $\beta$ & 6.73 days & 0.8 \\
${ }^{153} \mathrm{Sm}$ & $\beta$ & 46.50 hours & 1.1 \\
${ }^{186} \mathrm{Re}$ & $\beta, \mathrm{EC}$ & 3.72 days & 2.1 \\
${ }^{188} \mathrm{Re}$ & $\beta$ & 17.00 hours & 5.8 \\
${ }^{225} \mathrm{AC}$ & $\alpha$ & 10.00 days & 5.9 \\
${ }^{213} \mathrm{Bi}$ & $\alpha, \beta$ & 45.61 mins & 6.1 \\
${ }^{212} \mathrm{Bi}$ & $\alpha, \beta$ & 60.55 mins & 5.9 \\
${ }^{211} \mathrm{At}$ & $\alpha, \mathrm{EC}$ & 7.21 hours & 0.6 \\
${ }^{212} \mathrm{~Pb}$ & $\alpha, \beta$ & 10.64 hours & 5.8 \\
${ }^{223} \mathrm{Ra}$ & $\alpha$ & 11.44 days & 5.7 \\
${ }^{224} \mathrm{Ra}$ & $\alpha$ & 3.63 days & 6.0 \\
${ }^{227} \mathrm{Th}$ & $\alpha$ & 18.68 days &
\end{tabular}

$\alpha$ : Alpha, $\beta$ : Beta, EC: Electron capture, Y: Yttrium, I: Iodine, Lu: Lutetium, Sm: Samarium, Re: Rhenium, Ac: Actinium, Bi: Bismuth, At: Astatine, Pb: Lead, Ra: Radium, Th: Thorium

in the organs. The biodistribution of the radiopharmaceutical includes its transmission to organs, its uptake, metabolism, clearance, and excretion. The appropriate physical half-life for therapeutic radionuclides is between six hours and seven days. If we choose a radionuclide with a very short physical half-life, it will not be efficient and practical. If we choose a radionuclide with a long half-life, the exposure of the patient and the people around them will increase, since the dose absorbed in the patient's body is large. Having a very long physical half-life means that patients stay in the hospital for a long time, and the duration of isolation increases. This increases the cost of treatment. Information about the biological half-life depends on the pharmaceutical used. If the pharmaceutical remains in the patient's body too long, the physical half-life of the radionuclide should not be too long. Therefore, for effective radiopharmaceutical treatment, a radionuclide with a balanced biological and physical half-life should be selected $(8,10,22)$.

\section{Therapeutic radiopharmaceuticals used in breast cancer}

\section{Types of breast cancer}

Breast cancer is the most common type of cancer among women in the world and is a heterogeneous disease with different molecular subtypes. It is considered to be a mixture of four diseases: hormone receptor (HR)-positive and human epidermal growth factor 2 (HER2)-negative cancer; HR-positive and HER2-positive cancer; HR-negative and HER2-positive cancer and triple-negative cancer (23). In HR (+) tumors, cancer cells carry the receptors for hormones such as estrogen and progesterone. These hormones trigger the growth of HR (+) tumors. In HER2 (+) tumors, cancer cells overproduce a protein responsible for cell growth and proliferation called HER2/neu. Knowing these subtypes of breast cancer forms the basis of diagnosis cancer cells and are highly effective in therapies targeting ER-positive $(\mathrm{ER}+)$ breast cancers. ER + breast cancer is the most common subtype of breast cancer (25). Therapeutic approaches designed according to these subtypes in breast cancer treatment have increased interest in the potential for the use of radionuclide and radiopharmaceuticals in breast cancer treatment. These studies are included in this compilation.

\section{Therapeutic radiopharmaceuticals for breast cancer}

The exploration of breast cancer treatment beganin the $19^{\text {th }}$ century. Radiation therapy was applied in addition to surgical intervention in 1937 to protect the breast. The FDA approved the drug tamoxifen in 1978 for use in breast cancer treatment. In 1996, the FDA approved anastrozole as a breast cancer treatment that inhibits estrogen production. Another drug approved by the FDA in 1998 was trastuzumab, which targets HER2, which is overproduced by cancer cells. In 2006, raloxifene, which showed lowertoxicity than tamoxifen, was found to reduce the risk of breast cancer $(26,27)$. Targeted RT has been developed, and research continues to design new targeted radiopharmaceuticals using various FDA approved drugs.

New studies have gained momentum with the discovery of RT in addition to the known traditional methods used for breast cancer treatment. These new studies are based on radiopharmaceuticals formed by chelating a radioactive element (radionuclide) to a conjugate that targets tumor tissue (11). To give an example of one of these radiopharmaceuticals, in a preclinical breast cancer treatment study conducted by Luo et al. (28) in 2009, the breast cancer treatment drug trastuzumab (Herceptin) was conjugated with SOCTA and labeled with the radionuclide ${ }^{188}$ Re. ${ }^{188}$ Re-SOCTAtrastuzumab was administered intravenously to xenograft mice bearing BT-474 breast cancer overexpressing HER-2/neu.. They suggested that ${ }^{188} \mathrm{Re}$-SOCTA-trastuzumab was a potential candidate for radioimmunotherapy (28). To date, preclinical studies conducted 
in the last five years on radiopharmaceuticals used in breast cancer treatment are listed below.

Various nanoparticles (organic nanoparticles such as polymeric matrices and liposomes, as well as inorganic nanoparticles such as gold, metal oxides, and insoluble metal salts) are used as radioisotope carriers in RPT. Yook et al. (29) designed a new radiopharmaceutical for the treatment of triple-negative breast cancer (TNBC), in which epidermal growth factor receptors (EGFR) are overexpressed. Gold nanoparticles (AuNP; $30 \mathrm{~nm}$ ) were complexed with 1,4,7,10-tetraazacyclododecane1,4,7,10-tetraacetic acid (DOTA) conjugate to form a complex with emitter ${ }^{177} \mathrm{Lu}$ with polyethylene glycol (PEG) chains $(4 \mathrm{kDa})$. After this modification, they bound panitumumab (selectively binds to the epidermal growth factor receptor). EGFR-targeted AuNP ${ }^{177} \mathrm{Lu}-$ T-AuNP) and non-targeted ${ }^{177} \mathrm{Lu}-\mathrm{NT}$-AuNP were subcutaneously injected into CD-1 athymic mice bearing EGFR-positive MDAMB-468 human breast cancer tumors and imaging studies were conducted. It was concluded that ${ }^{177} \mathrm{Lu}-\mathrm{T}-\mathrm{AuNP}$ is a potential and promising application for TNBC therapy $(29,30)$.

Radioimmunotherapy represents the use of isotopes conjugated to monoclonal antibodies $(\mathrm{mAb})$ in therapy (31). Trastuzumab (Herceptin) is a monoclonal antibody that binds to HER2 receptors overexpressed on cancer cells and is covalently bound with 3-phosphonopropionic acid (CEPA) nanoparticles. This conjugate alpha emitter is marked with ${ }^{225}$ Ac. Superparamagnetic iron oxidebased nanoparticles (cipions) were used as the targeting conjugate $\left({ }^{225} \mathrm{Ac}^{\oplus} \mathrm{Fe}_{3} \mathrm{O}_{4}\right) \cdot{ }^{225} \mathrm{Ac}^{\oplus} \mathrm{Fe}_{3} \mathrm{O}_{4}$-CEPA-trastuzumab radio bioconjugate was shown to have high receptor affinity in in vivo studies and exhibit properties suitable for breast cancer treatment (32).

Morgenroth et al. (33) in their study evaluated the potential of the prostate-specific membrane antigen PSMA to target small molecules for radioligand therapy of TNBC. For in vivo experiments, nude mice were xenografted with MDA-MB231 estrogen receptor-expressing TNBC cells or MCF-7 breast cancer cells. In their therapeutic study with ${ }^{177} \mathrm{Lu}-\mathrm{PSMA}-617$, they demonstrated the potential of PSMA as a unique and specific targeting agent for TNBC.

Hernandez et al. (34) conducted a promising study by labeling tumortargeting alkyl phosphocholine (NM600) with ${ }^{177} \mathrm{Lu}$ radionuclide for targeted RT of TNBC. ${ }^{177} \mathrm{Lu}-\mathrm{NM} 600$ was administered toBALB/C mice carrying 4T07 and 4T1 mammary tumor grafts. This study suggested that the radiopharmaceutical ${ }^{177} \mathrm{Lu}-\mathrm{NM} 600$ is an appropriate therapy for targeted RT, and it was reported to show a favorable biodistribution (34).

Hagemann et al. (35) evaluated mesothelin (MSLN)-targeted ${ }^{227} \mathrm{Th}$ labeled conjugate (BAY2287411), which is an overexpressed membrane glycoprotein in breast cancer, in breast xenograft models produced from the patient. It was shown that this synthesized conjugate had in vivo and in vitro potential, and a dose-dependent regression in tumor growth was observed in treatment due to the increase in MSLN in breast cancer cells (35).

In their study, Yin et al. (25) created MCF-7 cell xenografts of ${ }^{131}$ I-fulvestrant in nude mice by radioiodine labeling fulvestrant (an endocrine treatment drug for breast cancer) and monitored tumor growth and critical organ function. The ${ }^{131} \mathrm{I}$-fulvestrant conjugate was determined to maintain its binding affinity to ER-positive $(\mathrm{ER}+)$ MCF-7 cells and exert a tumor suppressor effect on these cells. As a result, they noted that radioiodine labeling of fulvestrant was successful and could therefore be used to develop new drugs for breast cancer (25).

Thorek et al. (36) applied androgen receptor pathway activation to targeted alpha particle immunotherapy in breast cancer. To specifically treat breast cancer xenografts expressing androgen receptors, the antihK2 (hu11B6 antibody) alpha particle emitting ${ }^{225} \mathrm{Ac}$ radionuclide was labeled. Very promising results were obtained in aggressive androgen receptor-positive breast cancer cell lines with a ${ }^{225}$ Ac-hu11B6 radiopharmaceutical (36).

Gastrin-secreting peptide receptor (GRPr) is overexpressed in more than $75 \%$ of breast cancer cells. Gibbens-Bandala et al. (37) targeted ${ }^{177} \mathrm{Lu}-$ Bombesin $\left({ }^{177} \mathrm{Lu}-\mathrm{BN}\right)$ to GRPr and labeled it with paclitaxel (PTX), one of the main drugs in cancer therapy. In this study, MDAMB-231 breast cancer cells (GRPr-positive) were used. In vitro and in vivo studies of ${ }^{177} \mathrm{Lu}-\mathrm{BN}-\mathrm{PLGA}$ (PTX) showed high tumor uptake in MDA-MB-231 lesions. This makes it possible to monitor the progression of the disease with this conjugate.

Kasten et al. (38) investigated the potential of the ${ }^{212} \mathrm{~Pb}$-labeled antibody 225.28, which targets chondroitin sulfate proteoglycan 4 (CSPG4), in the treatment of TNBC in mouse models. ${ }^{212} \mathrm{~Pb}-225.28$ bound to CSPG4 with high affinity in TNBC cells and specifically inhibited its growth. These results support future therapeutic studies using ${ }^{212} \mathrm{~Pb}-225.28$ or other CSPG4-targeted radioimmunoconjugates, alone or in combinatorial approaches, against the TNBC model (38).

Costa et al. (39) investigated the effect of ${ }^{223} \mathrm{Ra}$ for the treatment of bone metastases in breast cancer patients. In light of this study, it was found that ${ }^{223} \mathrm{Ra}$ treatment can be used for the treatment of bone metastases in breast cancer patients. In a study conducted by Juzeniene et al. (40), the effects of alpha-emitting ${ }^{224} \mathrm{Ra}$ radionuclide treatment on osteolytic bone metastasis of MDA-MB-231 (SA)-GFP human breast cancer cells injected intracardially into mice were investigated. Treatment with ${ }^{224} \mathrm{Ra}$ solution was found to reduce the number of bone metastases. In light of these results, ${ }^{224} \mathrm{Ra}$ solution was proposed as a promising candidate for the treatment of breast cancer patients (40).

AuNP $(30 \mathrm{~nm})$ were labeled with ${ }^{177} \mathrm{Lu}$ radionuclide by binding to trastuzumab,PEG, or DOTA. ${ }^{177} \mathrm{Lu}$-AuNP-trastuzumab binds specifically to HER2-positive breast cells and enables effective radiopharmaceutical therapy (41). Cai et al. (42) linked $30 \mathrm{~nm}$ AuNP to trastuzumab with trastuzumab targeting HER2-positive breast cancer cells and ${ }^{111}$ In labeled with diethylenetriaminepentaacetic acid. The ${ }^{111}$ In-AuNP-trastuzumab radiopharmaceutical was shown to inhibit tumor growth without normal tissue toxicity for treatment in CD1 athymic mice (42).

\section{Conclusion}

Recent studies about the therapeutic efficacy of radiopharmaceuticals prepared using alpha and beta-emitting radionuclides for the treatment of breast cancer have shown significant potential. In the treatment of advanced breast cancer, radiolabeling the beta particle emitter ${ }^{177} \mathrm{Lu}$ radionuclide with nanoparticles and radioimmunotherapy with lowmolecular-weight protein ligands of the alpha emitters ${ }^{225} \mathrm{Ac}$ or ${ }^{213} \mathrm{Bi}$ appear promising due to the favorable pharmacokinetic properties of these radiopharmaceuticals. Radioiodine labeling of fulvestrant found that it can be used for breast cancer treatment. Other radioimmunoconjugates targeted to ${ }^{212} \mathrm{~Pb}-225.28$ or CSPG4 support therapy against the TNBC model. Local radiation therapy for HER2-positive breast cancer based 
on AuNP modified with trastuzumab and labeled with an auger electron emitter ${ }^{111}$ In was developed and was shown to arrest tumor growth without normal tissue toxicity. Enhancement of the radiopharmaceutical ${ }^{177}$ Lu-BN-PLGA (PTX) proved it to be suitable as a targeted paclitaxel delivery system with a concurrent radiotherapeutic effect for the treatment of GRPr-positive breast cancer. Additionally, ${ }^{223} \mathrm{Ra}$ and ${ }^{224} \mathrm{Ra}$ radionuclides were shown to have potential utility in the treatment of bone metastases in breast cancer patients.

In light of preclinical studies, radionuclides and radiopharmaceuticals used for breast cancer treatment represent an area of huge potential.

Peer-review: Externally peer-reviewed.

\section{Authorship Contributions}

Conception: B.A.G., F.Y.; Design: B.A.G., F.Y.; Data Collection and/or Processing: B.A.G., F.Y.; Analysis and/or Interpretation: B.A.G., F.Y.; Literature Search: B.A.G., F.Y.; Writing: B.A.G., F.Y.

Conflict of Interest: The authors declare no conflict of interest.

Financial Disclosure: The authors declare that this study received no financial support.

\section{References}

1. World Health Organization. Cancer Report, September 2018. Available from: https://www.who.int/health-topics/cancer\#tab=tab_1 [Crossref]

2. Mattiuzzi C, Lippi G. Current cancer epidemiology. J Epidemiol Glob Health 2019; 9: 217-222. (PMID: 31854162) [Crossref]

3. Kayano D, Kinuya S. Current consensus on ${ }^{131} \mathrm{I}-\mathrm{MIBG}$ therapy. Nucl Med Mol Imaging 2018; 52: 254-265. (PMID: 30100938) [Crossref]

4. Das S, Al-Toubah T, El-Haddad G, Strosberg J. ${ }^{177}$ Lu-DOTATATE for the treatment of gastroenteropancreatic neuroendocrine tumors. Expert Rev Gastroenterol Hepatol 2019; 13: 1023-1031. (PMID: 31652074) [Crossref]

5. Fendler WP, Rahbar K, Herrmann K, Kratochwil C, Eiber M. ${ }^{177} \mathrm{Lu}-$ PSMA radioligand therapy for prostate cancer. J Nucl Med 2017; 58: 1196-1200. (PMID: 28663195) [Crossref]

6. Naraev BG, Ramirez RA, Kendi AT, Halfdanarson TR. Peptide receptor radionuclide therapy for patients with advanced lung carcinoids. Clin Lung Cancer 2019; 20: e376-e392. doi: 10.1016/j.cllc.2019.02.007 (PMID: 30910575) [Crossref]

7. Mukherjee S, Ayanambakkam A, Ibrahimi S, Schmidt S, Charkrabarty $\mathrm{JH}$, Khawandanah M. Ibritumomab tiuxetan (Zevalin) and elevated serum human anti-murine antibody (HAMA). Hematol Oncol Stem Cell Ther 2018; 11: 187-188. (PMID: 29406240) [Crossref]

8. Tafreshi NK, Doligalski ML, Tichacek CJ, Pandya DN, Budzevich MM, El-Haddad G, et al. Development of targeted alpha particle therapy for solid tumors. Molecules 2019; 24: 4314. (PMID: 31779154)

9. Langbein T, Weber WA, Eiber M. Future of theranostics: an outlook on precision oncology in nuclear medicine. J Nucl Med 2019; 60: 13S-19S. doi: 10.2967/jnumed.118.220566 (PMID: 31481583) [Crossref]

10. Yeong $\mathrm{CH}$, Cheng $\mathrm{MH}, \mathrm{Ng} \mathrm{KH}$. Therapeutic radionuclides in nuclear medicine: current and future prospects. J Zhejiang Univ Sci B 2014; 15: 845-863. (PMID: 25294374) [Crossref]

11. Sgouros G. Radiopharmaceutical therapy. Health Phys 2019; 116: 175178. (PMID: 30585960) [Crossref]

12. Jadvar $H$. Targeted $\alpha$-therapy in cancer management: synopsis of preclinical and clinical studies. Cancer Biother Radiopharm 2020; 35: 475-484. (PMID: 32202923) [Crossref]
13. Williams WSC, Nuclear and particle physics. $1^{\text {st }}$ ed. Oxford: Oxford University; 1991. [Crossref]

14. Reed AB. The history of radiation use in medicine. J Vasc Surg 2011; 53(Suppl 1): 3S-5S. doi: 10.1016/j.jvs.2010.07.024 (PMID: 20869835) [Crossref]

15. Ferrier MG, Radchenko V, Wilbur S. Radiochemical aspects of alpha emitting radionuclides for medical application. Radiochim Acta 2019; 107: 1065-1085. [Crossref]

16. Sgouros G, Bodei L, McDevitt MR, Nedrow JR. Radiopharmaceutical therapy in cancer: clinical advances and challenges. Nat Rev Drug Discov 2020; 19: 589-608. (PMID: 32728208) [Crossref]

17. Ferrier MG, Radchenko V. An Appendix of radionuclides used in targeted alpha therapy. J Med Imaging Radiat Sci 2019; 50 (4 Suppl 1): S58-S65. doi: 10.1016/j.jmir.2019.06.051 (PMID: 31427258) [Crossref]

18. Uenak, P. Nuclear applications in life sciences. National Nuclear Science and Technologies Congress Proceedings Full Texts. 2009; 1: 387. [Crossref]

19. Gündoğdu EA, Özgenç E, Ekinci M, Özdemir ID, Aşıkoğlu M. Nükleer Tipta Görüntülemede ve Tedavide Kullanılan Radyofarmasötikler. Turkiye Klinikleri J Pharm Sci 2017. [Crossref]

20. Yong K, Brechbiel MW. Towards translation of ${ }^{212} \mathrm{~Pb}$ as a clinical therapeutic; getting the lead in! Dalton Trans 2011; 40: 6068-6076. (PMID: 21380408) [Crossref]

21. Ertay T. New radiopharmaceuticals and preclinical Imaging. Nuc Med Semin 2019: 5: 1-9. doi:10.4274/nts.galenos.2019.0001 [Crossref]

22. Jadvar $H$. Targeted $\alpha$-therapy in cancer management: synopsis of preclinical and clinical studies. Cancer Biother Radiopharm 2020; 35: 475-484. (PMID: 32202923) [Crossref]

23. Lakhtakia R, Burney I. A brief history of breast cancer: part III -tumour biology lays the foundation for medical oncology. Sultan Qaboos Univ Med J 2015; 15: 34-38. (PMID: 25685382) [Crossref]

24. Fisusi FA, Akala EO. Drug combinations in breast cancer therapy. Pharm Nanotechnol 2019; 7: 3-23. (PMID: 30666921) [Crossref]

25. Yin G, Zeng B, Peng Z, Liu Y, Sun L, Liu C. Synthesis and application of ${ }^{131}$ I-fulvestrant as a targeted radiation drug for endocrine therapy in human breast cancer. Oncol Rep 2018; 39: 1215-1226. (PMID: 29328488) [Crossref]

26. Akram M, Iqbal M, Daniyal M, Khan AU. Awareness and current knowledge of breast cancer. Biol Res 2017; 50: 33. (PMID: 28969709) [Crossref]

27. Healthline. History of Breast Cancer, April 2017, Available from: https:// www.healthline.com/health/history-of-breast-cancer [Crossref]

28. Luo TY, Tang IC, Wu YL, Hsu KL, Liu SW, Kung HC. Evaluating the potential of ${ }^{188} \mathrm{Re}$-SOCTA-trastuzumab as a new radioimmunoagent for breast cancer treatment. Nucl Med Biol 2009; 36: 81-88. (PMID: 19181272) [Crossref]

29. Yook S, Cai Z, Lu Y, Winnik MA, Pignol JP, Reilly RM. Radiation nanomedicine for egfr-positive breast cancer: panitumumab-modified gold nanoparticles complexed to the $\beta$-particle-emitter, (177)lu. Mol Pharm 2015; 12: 3963-3972. (PMID: 26402157) [Crossref]

30. Yook S, Cai Z, Lu Y, Winnik MA, Pignol JP, Reilly RM. Intratumorally injected 177lu-labeled gold nanoparticles: gold nanoseed brachytherapy with application for neoadjuvant treatment of locally advanced breast cancer. J Nucl Med 2016; 57: 936-942. (PMID: 26848176)

31. Goldenberg DM. Targeted therapy of cancer with radiolabeled antibodies. J Nucl Med 2002; 43: 693-713. (PMID: 11994535) [Crossref]

32. Cędrowska E, Pruszyński M, Gawęda W, Żuk M, Krysiński P, Bruchertseifer F, et al. Trastuzumab conjugated superparamagnetic iron oxide nanoparticles labeled with ${ }^{225} \mathrm{Ac}$ as a perspective tool for combined $\alpha$-radioimmunotherapy and magnetic hyperthermia of HER2-positive breast cancer. Molecules 2020; 25: 1025. (PMID: 32106568) [Crossref] 


\section{Altıparmak Güleç and Yurt. Breast Cancer Treatment}

33. Morgenroth A, Tinkir E, Vogg ATJ. Sankaranarayanan RA, Baazaoui F, Mottaghy FM. Targeting of prostate-specific membrane antigen for radioligand therapy of triple-negative breast cancer. Breast Cancer Res 2019; 21: 116. (PMID: 31640747) [Crossref]

34. Hernandez R, Grudzinski JJ, Aluicio-Sarduy E, Massey CF, Pinchuk AN, Bitton $\mathrm{AN}$, et al. ${ }^{177} \mathrm{Lu}-\mathrm{NM} 600$ targeted radionuclide therapy extends survival in syngeneic murine models of triple-negative breast cancer. J Nucl Med 2020; 61: 1187-1194. (PMID: 31862799) [Crossref]

35. Hagemann UB, Ellingsen C, Schuhmacher J, Kristian A, Mobergslien A, Cruciani V, et al. Mesothelin-targeted thorium-227 conjugate (MSLN-TTC): preclinical evaluation of a new targeted alpha therapy for mesothelin-positive cancers. Clin Cancer Res 2019; 25: 4723-4734. (PMID: 31064781) [Crossref]

36. Thorek DLJ, Ku AT, Mitsiades N, Veach D, Watson PA, Metha D, et al. Harnessing androgen receptor pathway activation for targeted alpha particle radioimmunotherapy of breast cancer. Clin Cancer Res 2019; 25 : 881-891. (PMID: 30254080) [Crossref]

37. Gibbens-Bandala B, Morales-Avila E, Ferro-Flores G, Santos-Cuevas C, Meléndez-Alafort L, Trujillo-Nolasco $\mathrm{M}$, et al. ${ }^{177} \mathrm{Lu}$-Bombesin-PLGA (paclitaxel): a targeted controlled-release nanomedicine for bimodal therapy of breast cancer. Mater Sci Eng C Mater Biol Appl 2019; 105: 110043. doi: 10.1016/j.msec.2019.110043 (PMID: 31546458) [Crossref]
38. Kasten BB, Oliver PG, Kim H, Fan J, Ferrone S, Zinn KR, et al. ${ }^{212} \mathrm{~Pb}$ labeled antibody 225.28 targeted to chondroitin sulfate proteoglycan 4 for triple-negative breast cancer therapy in mouse models. Int J Mol Sci 2018; 19: 925. (PMID: 29561763) [Crossref]

39. Costa RP, Tripoli V, Princiotta A, Murabito A, Licari M, Piazza D, et al. Therapeutic effect of ${ }^{223} \mathrm{Ra}$ in the management of breast cancer bone metastases. Clin Ter 2019; 170: e1-e3. doi: 10.7417/CT.2019.2100 (PMID: 30789190) [Crossref]

40. Juzeniene A, Bernoulli J, Suominen M, Halleen J, Larsen RH. Antitumor activity of novel bone-seeking, $\alpha$-emitting ${ }^{224} \mathrm{Ra}$-solution in a breast cancer skeletal metastases model. Anticancer Res 2018; 38: 1947-1955. (PMID: 29599310) [Crossref]

41. Cai Z, Yook S, Lu Y, Bergstrom D, Winnik MA, Pignol JP, et al. Local radiation treatment of HER2-positive breast cancer using trastuzumabmodified gold nanoparticles labeled with 177Lu. Pharm Res 2017; 34: 579-590. (PMID: 27987070) [Crossref]

42. Cai Z, Chattopadhyay N, Yang K, Kwon YL, Yook S, Pignol JP, et al. ${ }^{111}$ In-labeled trastuzumab-modified gold nanoparticles are cytotoxic in vitro to HER2-positive breast cancer cells and arrest tumor growth in vivo in athymic mice after intratumoral injection. Nucl Med Biol 2016; 43: 818-826. (PMID: 27788375) [Crossref] 